Cahiers $d u$ MONDE RUSSE

\section{Cahiers du monde russe}

Russie - Empire russe - Union soviétique et États indépendants

$51 / 4 \mid 2010$

Sciences humaines et sociales en Russie à l'Âge d'argent

\title{
Scott B. Smith, Captives of Revolution
}

\section{Thomas Chopard}

\section{(2) OpenEdition}

Journals

Édition électronique

URL : https://journals.openedition.org/monderusse/7376

DOI : 10.4000/monderusse.7376

ISSN : $1777-5388$

Éditeur

Éditions de l'EHESS

Édition imprimée

Date de publication : 25 novembre 2010

Pagination : 691-695

ISBN : 978-2-7132-2316-7

ISSN : $1252-6576$

Référence électronique

Thomas Chopard, «Scott B. Smith, Captives of Revolution », Cahiers du monde russe [En ligne], 51/4 I 2010, mis en ligne le 09 décembre 2011, consulté le 02 septembre 2022. URL : http://

journals.openedition.org/monderusse/7376; DOI : https://doi.org/10.4000/monderusse.7376

Ce document a été généré automatiquement le 2 septembre 2022

Tous droits réservés 


\title{
Scott B. Smith, Captives of Revolution
}

\author{
Thomas Chopard
}

\section{RÉFÉRENCE}

Scott B. SмIтн, Captives of Revolution. The Socialist Revolutionaries and the

Bolshevik Dictatorship, 1918-1923. Pittsburgh : University of Pittsburgh Press, 2011, $380 \mathrm{p}$.

1 En quelques mois, la littérature scientifique a produit presque autant d'ouvrages sur les socialistes-révolutionnaires (SR) qu'en quatre-vingts ans. Avec ce livre, Scott B. Smith expose les résultats de sa thèse qui porte sur le parti SR au cours de la guerre civile qui accompagna l'effondrement de l'Empire russe. Entamé au début des années 1990, ce travail a été nourri tout au long de la décennie par la publication de nouvelles sources. Les éléments nouveaux apportés par l'auteur - notamment par rapport aux travaux aujourd'hui classiques d'oliver Radkey ${ }^{1}$ - tiennent essentiellement dans l'approche: l'attention est portée non seulement sur les discours et les décisions politiques en tant que tels, mais aussi sur leurs interactions avec ceux des autres acteurs de la guerre civile. Scott B. Smith analyse les catégories de discours, leurs sources de légitimation et replace ceux des SR au confluent du discours de classe et du discours nationaliste. Se réclamant des travaux de Pierre Bourdieu, l'ouvrage a donc pour objectif, en mettant en lumière l'ambivalence des théories du parti SR, de dégager la captation du discours révolutionnaire par les bolcheviks, qui se positionneraient ainsi comme la seule alternative à la contre-révolution. Cette captation est pour l'auteur de première importance : elle vient en effet définir l'identité sociale des combattants et structure pour une large part la signification de la violence déployée pendant la guerre civile. Plus encore, elle serait au cœur de ce que l'on appelle une "culture de guerre civile », qui s'accomplirait dans le procès final des SR en 1922 et dont le stalinisme serait l'héritier. 
2 Afin de décrire cet effet de captation et l'impossibilité du parti SR à exister comme alternative autonome, Smith suit chronologiquement l'évolution de ce parti au cours de la guerre civile. Mais l'auteur se concentre surtout sur l'année 1918 (ch. I à V), qu'il considère comme « l'année décisive de la guerre civile » : de fait, il s'agit surtout de celle durant laquelle les SR participent à deux gouvernements antibolcheviks - le Comité des membres de l'Assemblée constituante (Komuč) sur la Volga, qu'ils contrôlent (ch. III), et le Gouvernement provisoire sibérien, dont ils seront écartés (ch. IV).

3 L'ouvrage propose d'emblée des positions solides et une méthode stimulante. Cette dernière consiste à déployer l'ensemble des possibles qui s'offrent au parti SR et qui sont discutés en son sein : opportunités d'alliance (essentiellement avec les bolcheviks), de rhétorique («de classe» ou nationaliste), d'actions, etc., soit un certain nombre d'hypothèses que l'auteur pose comme autant de «dilemmes». Ce choix méthodologique a le mérite d'intégrer le parti SR à l'ensemble des événements de la guerre civile et d'engager des comparaisons avec les autres partis. Il présente aussi une limite : il tend à écrire l'ensemble de l'histoire du parti SR comme étant dictée par des choix cruciaux dictés d'en haut. Congrès du parti, réunions, programmes, etc., tiennent donc une place centrale dans l'ouvrage. Une « histoire sociale » serait entravée par le manque de sources (p.XV); l'esquisser aurait toutefois pu offrir un contrepoids à ce schéma général.

4 Le premier postulat défendu par l'auteur concerne le début de la guerre civile. Smith le date de la formation de la Constituante (suivie de sa dissolution quasi immédiate). Il se fonde sur les journaux contemporains qui commencent effectivement à employer massivement l'expression de "guerre civile", mais considère toutefois que la concomitance d'une rhétorique politique et d'une série d'événements violents est nécessaire pour parler de guerre civile. Or, cette rhétorique s'amorce bien chez les SR autour de la Constituante. La guerre civile apparaît donc ici non seulement comme une guerre, mais aussi comme un combat politique. On pourrait arguer que l'expression apparait dès septembre dans les journaux et que le phénomène de violences endémiques au sein de l'ancien Empire date de la dissolution de l'armée tsariste, mais il est indéniable que l'opposition politique aux bolcheviks n'apparait clairement qu'en janvier.

5 Le premier dilemme mis en lumière par l'auteur concerne l'adoption d'un discours politique centré soit sur la "classe", dans la continuité révolutionnaire, soit sur la nation, avec une insistance sur la patrie en danger dans la lignée de la Première Guerre mondiale. Cette alternative se pose à deux reprises: lors de la dissolution de la Constituante et lors du traité de Brest-Litovsk. Smith note, dans un premier temps, l'indécision des SR lors de la dissolution de la Constituante; et, dans un second, leur adoption d'une rhétorique nationaliste à la faveur du traité.

6 La dissolution les amène en effet à se demander s'ils doivent combattre le nouveau pouvoir bolchevik au nom de la Constituante ; l'auteur montre toutefois que leur culture révolutionnaire, essentiellement centrée sur la Révolution française, leur fait craindre une réaction violente contre le pouvoir révolutionnaire (quel qu'il soit), assimilée à un nouveau Thermidor. Ils commencent donc par choisir une opposition interne au régime soviétique; les violences en province (bunty, dissolution de conseils à minorité bolchevique, etc.) montrent néanmoins un glissement progressif vers un soutien aux formes de protestation antibolcheviques.

7 C'est le traité de Brest-Litovsk qui radicalise l'opposition des SR en véritable combat armé. Acté lors de son VIII ${ }^{e}$ Congrès - et analysé comme une alliance entre Allemands et 
bolcheviks -, le traité réactive au sein du parti SR un discours nationaliste hérité du premier conflit mondial: la lutte contre les bolcheviks devient lutte contre les Allemands et défense de la patrie envahie, le parti se transformant en «parti combattant» (p. 54). Smith prête une attention toute particulière à la tentative de mobilisation des biens et des personnes par les SR dans les régions déjà les plus hostiles au pouvoir bolchevik: Volga et Sibérie. L'auteur montre bien l'impéritie des SR à mobiliser autour de la question nationale dans ces deux régions. La question du ravitaillement, cruciale au cours de la guerre civile, est abordée notamment à travers la description des insurrections "vertes". De même, les tentatives SR de fédérer les différents pôles antibolcheviks en un pouvoir panrusse unique, appuyé sur la légitimité de la Constituante dissoute, sont étudiées, notamment par le biais de la concurrence entre les SR et l'Union pour la régénération de la Russie, antirévolutionnaire, qui aboutit à l'éviction des SR et enfin au coup d'État militaire de l'amiral Kolčak en Sibérie. Conseils du parti, réunions, négociations sont exposés avec précision, mais le propos aurait pu être plus aiguisé, en faisant davantage jaillir les lignes de force.

8 L'autre forme de radicalisation de la lutte SR consiste dans la reprise du terrorisme visant tout à la fois bolcheviks et Allemands au cours de l'année 1918. L'auteur montre comment le parti SR, tout en condamnant la reprise des activités terroristes héritées de l'ancien régime, laisse les cellules opérer sans les inquiéter autrement. Si la tentative d'assassinat de Lenin par Fanny Kaplan est largement connue, l'historien revient, à travers des sources testimoniales et judiciaires, sur la constitution de la cellule centrée autour de Semenov, à l'origine de leur premier attentat contre Volodarskij, rédacteur en chef de Krasnaja Gazeta, le 20 juin 1918. Par le croisement des sources, Smith permet de façon convaincante de conclure à la responsabilité, sinon unique à tout le moins majeure, de Fanny Kaplan dans l'attentat contre Lenin à l'origine du déclenchement de la Terreur rouge.

9 Tout comme le traité de Brest-Litovsk, l'échec des tentatives pour constituer un pouvoir antibolchevik tant sur la Volga (reprise par les Rouges) qu'en Sibérie (où les SR sont évincés par Kolčak), pousse le parti SR à modifier son discours qui ne peut plus, faute d'Allemands, s'appuyer sur une rhétorique nationaliste. S'impose alors définitivement un discours qui aborde la guerre civile en tant que lutte des classes et oblige les SR à repenser la place des bolcheviks au sein du mouvement révolutionnaire. Les SR disposent ainsi de trois options au cours de l'année 1919: aider les bolcheviks contre les Blancs, assimilés à la contre-révolution; accepter le parti bolchevik afin de reconstruire leur propre parti au sein des organes soviétiques; renverser le pouvoir blanc afin de recouvrer une position susceptible d'accélérer la fin de la guerre civile. Leurs errances suite aux défaites de 1918 sont bien mises en évidence: le parti bolchevik prend ainsi alternativement la tournure d'un partenaire potentiel, d'un pouvoir temporairement vainqueur ou d'un ennemi à combattre. De fait, toutes les options SR conduisent à des échecs: la tentative de renverser le pouvoir blanc de Sibérie, en s'appuyant sur des instances locales et des militaires prorévolutionnaires, est notamment analysée presque au jour le jour jusqu'à son écrasement et à la dissolution des corps armés pro SR en troupes partisanes qui accélèrent l'effondrement du front de Kolčak malgré le refus d'une alliance par les bolcheviks.

10 L'année 1920 marque les derniers faits d'armes des SR au cours de la guerre civile. Smith analyse, à la lumière des nouveaux matériaux disponibles, leur participation réelle (largement fantasmée par les bolcheviks) aux mouvements paysans, notamment dans la 
province de Tambov, et plus encore la répression qui suit, avec - pour les SR - des arrestations et un exil massifs. Pour ces dernières années de la guerre civile, marquées surtout par les désordres des campagnes, l'auteur met en évidence la mauvaise appréciation de la situation par un parti SR très largement affaibli. Cette erreur d'interprétation repose très largement, selon lui, sur les catégories héritées de la culture révolutionnaire d'ancien régime, inopérante dans le cadre d'une guerre et d'une mobilisation matérielle et idéologique de masse, et n'ayant pas pris en compte les mutations apportées par la révolution au parti bolchevik et à son mode de gouvernement.

11 Mais c'est le procès des SR de 1922 qui est emblématique de la répression finale du parti : le chapitre VII mène à son point culminant l'hypothèse centrale de l'ouvrage qui fait de la répression bolchevique contre les SR la matrice des pratiques répressives soviétiques. Smith insiste, davantage encore que Sheila Fitzpatrick qu'il discute, sur cette dimension formatrice des années de guerre civile (essentiellement pour lui l'année 1918): l'expérience de guerre, et surtout sa mise en scène (à travers les procès spectacles comme celui des SR), ainsi que, plus tard, les séances d'autocritique, seraient cruciales dans l'autodéfinition du parti bolchevik et de ses membres.

Les SR seraient donc les pionniers d'une longue lignée de procès contre les ennemis intérieurs, dont seuls les effets et l'environnement varieraient au fil des ans (p. 276). Le procès de 1922 en lui-même est largement étudié, tant dans sa dimension judiciaire (acte d'accusation, stratégies de défense, etc.) que dans tout ce qui l'entoure: campagne d'agitation, pressions de la part des dirigeants soviétiques pour hâter sa conclusion. Cette finesse d'analyse aurait toutefois pu ouvrir sur deux aspects qui semblent négligés : le rôle même du procès dans la société soviétique et une comparaison avec les autres procès spectacles de l'ère stalinienne. Malgré la précision de la description, le rôle symbolique du procès n'est en effet jamais analysé : tandis que les SR usent dans leur défense d'une rhétorique révolutionnaire dans la lignée de la subversion de 1917, le pouvoir soviétique insiste au contraire sur la responsabilité individuelle des accusés, ne mettant pas en avant des actes collectifs d'insurrection (Tambov est ainsi largement passé sous silence), mais plutôt des actes solitaires (notamment le terrorisme) qui, d'un point de vue symbolique, empêchent le retour à la normale, à la paix, à l'ordre. Désir qui transparaît dans les arguments soviétiques. Quant à une possible comparaison, l'accent mis sur les faits, tant dans la propagande que par le procureur Krylenko, présente une rupture avec la criminalisation des intentions, des projets et des opinions qui caractériseront les procès staliniens. Ce procès est effectivement un procès politique mais il n'est pas pour autant un procès monté de toutes pièces. En ce sens, plutôt que de le tirer uniquement du côté des procès de Šahty et de Moscou, une comparaison avec les autres procès qui closent la guerre civile aurait favorisé une meilleure appréhension de la spécificité (encore à démontrer) du procès des SR. Cette mise en contexte aurait permis d'en saisir justement l'ambivalence : procès spectacle - matrice des procès staliniens à venir et tout à la fois procès d'épuration, typique des sorties de guerre.

13 L'analyse finalement trop descriptive du procès empêche aussi l'auteur d'envisager les silences de l'accusation, tout aussi importants dans "l'expérience formatrice » de la guerre civile que la figure du «traître au socialisme ». La participation massive des SR aux insurrections paysannes, dont les bolcheviks sont pourtant persuadés, n'est jamais mise en avant par Krylenko. Se dessine en creux le silence qui accompagne cette guerre du pouvoir soviétique contre les campagnes. Une guerre qui, en 1922, bat encore son 
plein et que Smith ne mentionne aucunement. L'héritage de cette "sale guerre" (Nicolas Werth), sa réduction au silence, une représentation des campagnes comme toujours susceptibles de chercher à se soustraire à l'autorité soviétique, sont pourtant voués à peser lourd sur les pratiques soviétiques, notamment au moment de la collectivisation.

14 Ces réserves tiennent au final plus aux prétentions particulièrement élevées de l'ouvrage qu'à son contenu réel qui vient renouveler, de façon extrêmement fouillée et stimulante, les travaux sur l'opposition précoce au bolchevisme. Si tout dire de la guerre civile et de la formation du pouvoir soviétique à travers les SR ne semble pas possible, presque tout ce qui concerne les SR eux-mêmes a été présenté dans ce travail.

\section{NOTES}

1. Pour la même période que le présent ouvrage, citons notamment, parus chez Columbia University Press en 1958 et 1963 : 1) The Agrarian Foes of Bolshevism: Promise and Default of the Russian Socialist Revolutionaries, February to October 1917 ; 2) The Sickle under the Hammer: the Russian Socialist Revolutionaries in the Early Months of Soviet Rule. 